\title{
A Combinatorial Optimization Approach for the Electrical Energy Management in a Multi-Source System
}

\author{
Yacine Gaoua ${ }^{1,2,3}$, Stéphane Caux ${ }^{1}$ and Pierre Lopez $^{2,3}$ \\ ${ }^{1}$ LAPLACE UMR 5213 CNRS, INPT, UPS, 2 rue Camichel, F-31071 Toulouse, France \\ ${ }^{2}$ CNRS, LAAS, 7 avenue du colonel Roche, F-31400 Toulouse, France \\ ${ }^{3}$ Univ de Toulouse, LAAS, F-31400 Toulouse, France \\ \{gaoua, caux\}@laplace.univ-tlse.fr, \{gaoua, lopez\}@laas.fr
}

\begin{abstract}
Keywords: Energy Management: Modeling: Combinatorial Optimization: Off-line Optimization: Dynamic Programming: Quasi-Newton Method: Branch-and-Cut Method: Operating Point: Energy Losses: Linearization.

Abstract: $\quad$ Minimizing the consumption of hydrogen by a fuel cell system in a hybrid vehicle can reduce its environmental impact and increase its autonomy. However an intelligent management of power distribution is essential to meet the demand of the powertrain. The characteristics of the sources constituting the energy chain of the hybrid vehicle (efficiency and energy losses) make the mathematical model nonlinear. Solution methods such as Dynamic Programming and Quasi-Newton which have so far been developed in previous works give satisfactory results but with very large computation times. In this paper, a new combinatorial model is proposed and a Branch-and-Cut method is developed to solve the problem to optimality. This approach leads to drastically reduced computation times.
\end{abstract}

\section{INTRODUCTION}

Hybrid vehicles use at least two energy sources to fuel their engines. The energy chain of the vehicle concerned is composed of a Fuel Cell System (FCS) which uses hydrogen to produce electrical energy through the chemical reaction with oxygen, supercapacitors for energy storage characterized by their energy losses, and an electric motor (powertrain). The challenge is to intelligently manage the power distribution by the two energy sources to meet the demand of the powertrain with the goal of minimizing the consumption of hydrogen by the FCS while respecting operational and safety constraints (Bernard et al., 2010) (Caux et al., 2011). Several methods and approaches such as dynamic programming (Brahma et al., 2000) using Bellman principle, or the quasiNewton method (Guemri et al., 2012) have been developed on this subject. These methods give suboptimal results with very large computing time due to the complexity of the underlying nonlinear problem and discretization required. The objective of this study is to improve the results obtained in previous work and provide a decision as quickly as possible to start the mission.

In the first part of this paper, the necessary background is given to highlight the model and solution approaches developed in previous works. The second part presents the new model and an application of the Branch-and-Cut method on the problem. Finally a third part is dedicated to the presentation of results to evaluate the performance of the proposition.

\section{THE ENERGY CHAIN}

The energy chain is composed of a FCS connected to the electric bus by an unidirectional converter, a pack of supercapacitors connected in series and in parallel to store energy which is also connected to the bus via a bidirectional converter. The supercapacitor provides energy when the vehicle is in traction and stores it when the vehicle brakes (principle of the transformation of kinetic energy into electrical energy).

The converter is an electronic power module which generates a regulated output voltage. It serves to maintain the bus voltage to its reference, despite the power demands of the electric motor and changes in voltage of the FCS and the supercapacitor. Its efficiency is often very high ranging from $93 \%$ to $97 \%$.

In reality, the FCS consists of the fuel cell itself and its ancillaries (air compressor, pumps of temperature control and humidification). The power absorbed 
by the air compressor depends on the power provided by the FCS which represents $80 \%$ of the total power consumed by the ancillaries.

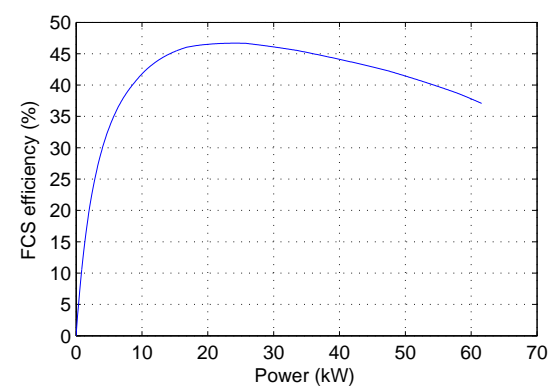

Figure 1: FCS efficiency.

Simulations and experiments (Caux et al., 2011) showed that the maximum efficiency of the FCS used with controlled pressure, temperature and humidity is equal to $46.53 \%$ for a power of $22.28 \mathrm{~kW}$, as shown in Figure 1.

\section{MATHEMATICAL FORMULATION}

To minimize the hydrogen consumption by the FCS off-line, a mission profile provided by INRETS (National REsearch Institute on Transport and their Security) which corresponds to the instantaneous demand required by the electric motor $P_{\text {req }}$ of a hybrid vehicle in urban area on a period of $T=560 \mathrm{~s}$, is given in Figure 2.

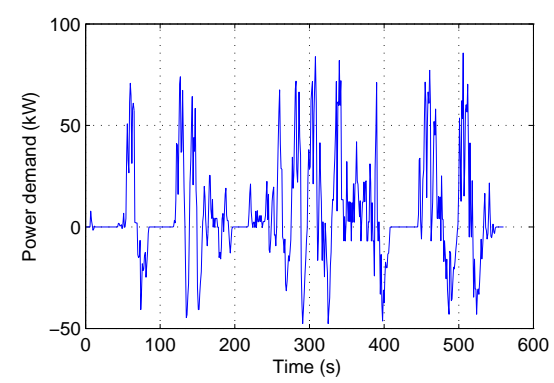

Figure 2: INRETS Mission profile.

The objective function is to minimize the hydrogen consumption $P_{h}$, modeled as:

$$
\text { Minimize } \sum_{t=1}^{T} P_{h}(t) \Delta t
$$

where $\Delta t$ is a sampling time between two consecutive instants.

The objective function can also be written in terms of FCS efficiency $\eta_{f c s}$ whose function is determined by a polynomial approximation and power supplied $P_{f c s}$, as shown in Equation 2. The nature of the FCS efficiency function makes the objective function nonlinear (Figure 1).

$$
\text { Minimize } \sum_{t=1}^{T} \frac{P_{f c s}(t)}{\eta_{f c s}\left(P_{f c s}(t)\right)} \Delta t
$$

The power provided by the FCS $P_{f c s}$ and the supercapacitor $P_{s e}$ must meet the demand of the electric motor when it is in traction, defined in Equation 3.

$$
P_{f c s}(t)+P_{s e}(t)=P_{r e q}(t)
$$

Forcing the supercapacitor to recover all braking energy can sometimes lead the FCS to work with a poor efficiency, hence the importance of Constraint 4.

$$
P_{\text {req }}(t) \leq P_{\text {se }}(t) \leq 0
$$

The FCS is seen as a production source. It can provide a maximum power of $P_{f c s}^{\max }$ (see Table 1 ) without recovering in braking phases.

$$
0 \leq P_{f c s}(t) \leq P_{f c s}^{\max }
$$

The advantage of the supercapacitor is that it can provide power and recover braking energy in the limit of its capacity defined in Equation 6:

$$
P_{s e}^{\min } \leq P_{s e}(t) \leq P_{s e}^{\max }
$$

For energy storage, the state of charge of the supercapacitor $S O C_{s e}$ at a given time is calculated based on the previous state of charge and power provided during this period of time:

$$
S O C_{s e}(t)=S O C_{s e}(t)-P_{s}(t) \Delta t
$$

The state of charge of the supercapacitor at each time must not exceed its storage capacity defined by an upper and lower bound $\left(S O C_{s e}^{\max }, S O C_{s e}^{\min }\right)$ :

$$
S O C_{s e}^{\min } \leq \operatorname{SOC}_{s e}(t) \leq S O C_{s e}^{\max }
$$

Table 1: Input parameters of the model.

\begin{tabular}{|c|c|}
\hline Parameter & Value \\
\hline$S O C_{s e}(0)$ & $900 \mathrm{~kW} . s$ \\
\hline$S O C_{s e}^{\min }$ & $400 \mathrm{~kW} . s$ \\
\hline$S O C_{s e}^{\max }$ & $1600 \mathrm{~kW} . \mathrm{s}$ \\
\hline$P_{s e}^{\min }$ & $-60 \mathrm{~kW}$ \\
\hline$P_{s e}^{\max }$ & $60 \mathrm{~kW}$ \\
\hline$P_{f c s}^{\max }$ & $70 \mathrm{~kW}$ \\
\hline$T$ & $560 \mathrm{~s}$ \\
\hline$\Delta t$ & $1 \mathrm{~s}$ \\
\hline$I_{f c s}$ & 601 \\
\hline$J_{s e}$ & 2 \\
\hline
\end{tabular}


By integrating the energy losses of the supercapacitor, the following constraint is imposed

$$
P_{s}(t)=P_{s e}(t)+\operatorname{Loss}_{s e}\left(P_{s e}(t)\right)
$$

where $P_{S}$ is the real power supplied or recovered by the supercapacitor and $\operatorname{Loss}_{s e}$ its energy losses function as shown in Figure 3.

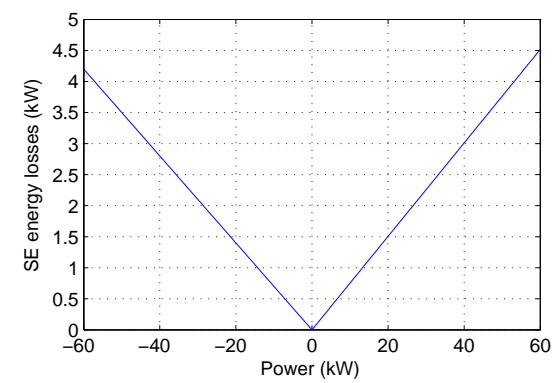

Figure 3: Energy losses of the supercapacitor.

To allow the sequence of tasks in off-line optimization without charging the supercapacitor artificially at the end of each mission, a condition is imposed on its final state of charge

$$
S O C_{s e}(T)=S O C_{s e}(0)
$$

where $S_{S C}(0)$ is the initial state of charge of the supercapacitor.

\section{BACKGROUND ON SOLVING METHODS}

In this section, the solution methods previously developed and applied to the energy management model are presented.

\subsection{Dynamic Programming}

Dynamic programming is a sequential combinatorial optimization method for finding the optimal solution using the Bellman principle: A sequence of decisions is optimal if, regardless of the point considered, subsequent decisions are a result of optimal decisions for the sub-dynamic problem with this point as starting. The discretization of the time horizon and the energy space of the storage element (supercapacitor) are required to apply dynamic programming on the model of the electric power management (Pérez et al., 2006).

The weakness of the application of dynamic programming on the model of power management, is related to the discretization of the supercapacitor energy space in $N$ points of $\Delta E$ stepsize. Indeed, increasing

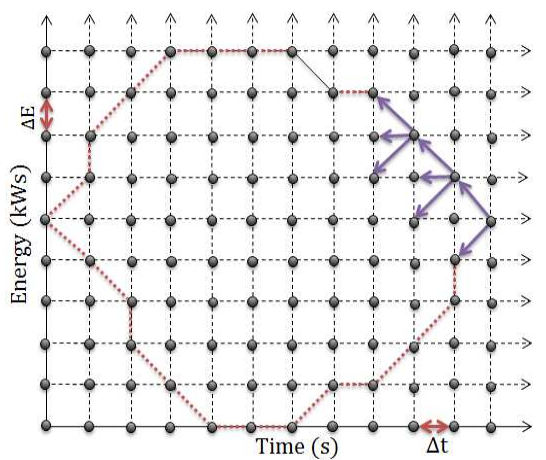

Figure 4: Dynamic Programming principle.

the number of possible states of charge of the supercapacitor causes more choices and calculations to determine the optimal sequence that minimizes the criterion of hydrogen consumption. For a discretization of the time horizon in $\Delta t=1 s$ and the energy space of the supercapacitor in $1 \mathrm{~kW} . s$, the optimal solution is $10131 \mathrm{~kW} . s$, which corresponds to the hydrogen consumed by the FCS on INRETS profile with a computation time of 22 hours. By decreasing the stepsize of the supercapacitor energy space energy, the solution will be improved but the computing time explodes.

\subsection{Quasi-Newton Method}

In numerical optimization, Quasi-Newton algorithm is an iterative method for solving nonlinear problems by using Karush-Kuhn-Tucker conditions and the calculation of the Hessian and the second derivative of the Lagrangian. The local minimum is found when the gradient is zero. The solution found by this method using fmincon function integrated in Matlab Optimization toolbox, is a local optimum. After the polynomial approximation of the two nonlinear functions (FCS efficiency and supercapacitor energy losses) by polynomials of degree 15 in order to reduce errors of approximations, the consumption of hydrogen used by the FCS is $8750 \mathrm{~kW} . s$, with a computation time of $23 \min$ (Guemri et al., 2012).

\section{COMBINATORIAL MODELING}

In the previous model, the objective function and the function of the energy losses of the supercapacitor are nonlinear and this makes finding an optimal solution not guaranteed. The principle of this new modeling is to work with the original data without using the approximations of the objective function and the energy losses function. 
Consider the operating point of the FCS $i$ characterized by its efficiency $\eta_{f c s}(i)$ and its energy supplied $P_{f c s}(i)$, and $I_{f c s}$ the number of operating points of the fuel cell system.

The decision variables used in the combinatorial model are:

- $X(t, i)$ : Binary variables, activation or not of the operating point $i$ at time $t$

- $Y(t, j)$ : Binary variables, activation or not of the losses energy equation $j$ at time $t$,

- $P_{s e}(t)$ : Power supplied or recovered by the supercapacitor at time $t$,

- $S O C_{s e}(t)$ : State Of Charge of the supercapacitor at time $t$,

- $\operatorname{Elos}_{s e}(t)$ : Energy losses by the supercapacitor at time $t$.

By integrating the operating points in the model, the objective function becomes:

$$
\sum_{t=1}^{T} \sum_{i=1}^{I_{f c s}} X(t, i) \frac{P_{f c s}(i)}{\eta_{f c s}(i)} \Delta t
$$

The capacity constraint of the FCS (Equation 5) is removed, and the satisfaction demand constraint when the vehicle is in traction (Equation 3) is replaced by:

$$
P_{s e}(t)+\sum_{i=1}^{I_{f c s}} X(t, i) P_{f c s}(i)=P_{r e q}(t)
$$

knowing that one FCS operating point is actived at each time $t$ :

$$
\sum_{i=1}^{I_{f c s}} X(t, i)=1
$$

Supercapacitor energy losses function is piecewise linear convex function (Figure 3 ) and can be written as:

$$
\operatorname{Elos}_{s e}(t)=\alpha_{j} P_{s e}(t)+\beta_{j}, P_{s e}(t) \in\left[\gamma_{j}, \gamma_{j}^{\prime}\right]
$$

where $\alpha_{j}$ is the gradient of the line $j$ and $\beta_{j}$ its intercept over the interval $\left[\gamma_{j}, \gamma_{j}^{\prime}\right]$

To avoid the polynomial approximation, a formulation using the max function is presented:

$$
\operatorname{Elos}_{s e}(t)=\max _{j=1}^{J_{s e}} \alpha_{j} P_{s e}(t)+\beta_{j}
$$

where $J_{s e}$ is the number of linear functions and $j \in J_{s e}$ its index. This function can also be modeled as linear equations system using binary variables and a big- $M$ constant:

$$
\begin{gathered}
\operatorname{Elos}_{s e}(t) \leq \alpha_{j} P_{s e}(t)+\beta_{j}+M(1-y(j, t)) \\
\operatorname{Elos}_{s e}(t) \geq \alpha_{j} P_{s e}(t)+\beta_{j} \\
\sum_{j=1}^{J_{s e}} y(j, t)=1
\end{gathered}
$$

The energy losses constraint (Equation 9) is replaced by:

$$
P_{s}(t)=P_{s e}(t)+E \operatorname{Los}_{s e}(t)
$$

\section{SOLVING AND SIMULATIONS}

The branch-and-cut algorithm (Winston, 1994) used to solve the model of energy management is an exact hybrid method for combinatorial optimization. It integrates cutting planes and branch and bound methods. This method solves NP-hard problems effectively.

The principle of this method is to solve the relaxation of the integer linear problem using Simplex algorithm. If the solution $X^{*}$ found is feasible for the integer linear problem then, this solution is optimal; if not, a cutting plane method is applied (Papadimitriou and Steiglitz, 1982). The cutting plane method consists of iteratively adding cuts violated by the solution of the relaxed problem until no further cuts are violated. The purpose of this method is to try to find an integer optimal solution or reduce the domain of non-integer values to start the branch-and-bound algorithm (Rardin, 1998).

The branch-and-bound method is used to solve the linear integer problem by separating the relaxed problem into two subproblems and evaluating their solutions. The separation principle is to choose a noninteger variable in the optimal solution of the relaxed problem and separate it into two sub-problems by adding the constraint $x_{i} \leq\left\lfloor x_{i}^{*}\right\rfloor$ to the first subproblem, the constraint $x_{i} \geq\left\lceil x_{i}^{*}\right\rceil$ to the second, and solve them with the Simplex method. This process is repeated until the optimal solution is found.

For solving, an exact branch-and-cut method is applied using IBM - Ilog Cplex 12.4 and the optimal solution was found, as shown in the following figures. The consumption of hydrogen used by the fuel cell system is $8750 \mathrm{~kW} . \mathrm{s}$ with a computation time of 2.59 $s$.

The FCS supply power to the electric motor, maintains storage of the supercapacitor between its bounds and recharges to its initial level at the end of the mission. As shown in Figure 5, the FCS is generally used 


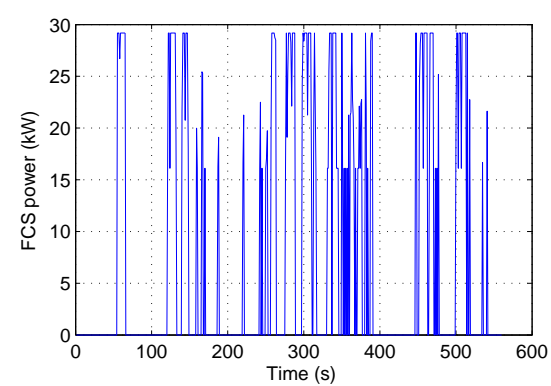

Figure 5: Power provided by the FCS.

with high efficiency to minimize energy losses and this explains the low consumption of hydrogen.

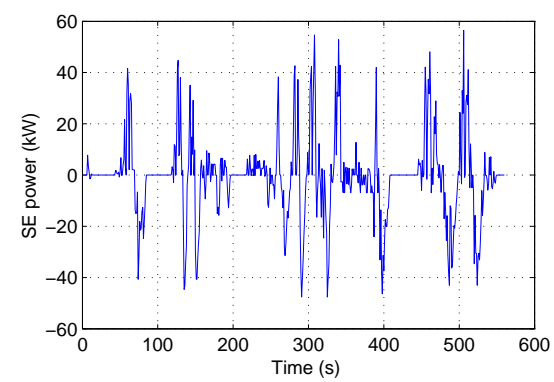

Figure 6: Power provided/recovered by the SE.

The supercapacitor recovers power to supply it when the vehicle is in traction (see Figure 6). Sometimes recovering all the braking energy is not a good strategy because it forces the FCS to supply the rest of the required power with low efficiency involving energy losses.

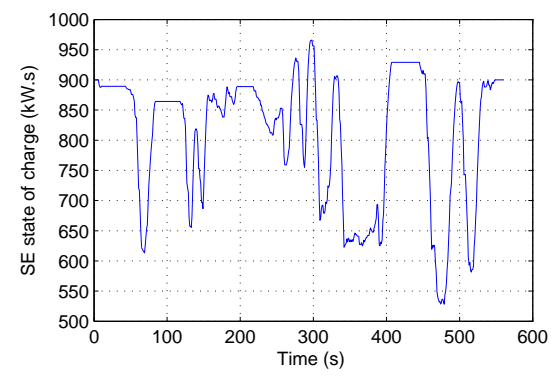

Figure 7: State of charge of the SE.

In Figure 7, the storage capacity is respected and the supercapacitor is reloaded to its original capacity at the end of the mission.

\section{CONCLUSION}

The aim of managing distribution of electrical energy is to meet the demand of the electric motor and also to minimize the consumption of hydrogen by the fuel cell. The methods previously developed can find satisfactory solutions (suboptimal) but with very large computation times. In reality, to start a multi-source system such as a hybrid vehicle on a known trajectory or mission profile, it is important to give quick decisions to manage its energy distribution. The new combinatorial model is efficient and it can find the optimal solution of the problem in a very short time.

\section{REFERENCES}

Bernard, J., Delprat, S., Guerra, T. M., and Büchi, F. N. (2010). Fuel efficient power management strategy for fuel cell hybrid powertrains. Control Engineering Practice, 18(4):408-417.

Brahma, A., Guezennec, Y., and Rizzoni, G. (2000). Optimal energy management in series hybrid electric vehicles. In Proceedings of the American Control Conference, pages 60-64, Chicago, 1llinois, USA.

Caux, S., Wanderley-Honda, D., Hissel, D., and Fadel, M. (2011). On-line energy management for HEV based on particle swarm optimization. The European Physical Journal Applied Physics, 54:1-9.

Guemri, M., Caux, S., and Ngueveu, S. U. (2012). Using quasi-Newton method for energy management in electrical multi source systems. In 11th International Conference on Environment and Electrical Engineering (EEEIC), pages 194-199, Venice, Italy.

Papadimitriou, C. H. and Steiglitz, K. (1982). Combinatorial Optimization: Algorithms and complexity. Prentice-Hall.

Pérez, L. V., Bossio, G. R., Moitre, D., and García, G. O. (2006). Optimization of power management in an hybrid electric vehicle using dynamic programming. Mathematics and Computers in Simulation, 73:244254.

Rardin, R. L. (1998). Optimization in operations research. Prentice-Hall.

Winston, W. L. (1994). Operations research: Applications and algorithms. Wadsworth. 